\title{
Nichtinvasive Ventilation in der Akutmedizin: Wer, wann, wie lang? R. Ragette (oder „das zweischneidige Schwert")
}

Noninvasive Ventilation in Critical Care: Who, When, How Long? Friend or Foe?

Mit nicht-invasiver Ventilation (NIV) bezeichnet man den Einsatz von maschineller positiver Druckbeatmung auf nichtinvasive Weise, d. h. über Nasen-, Nase-Mund- oder Ganzgesichtsmaske und unter Umgehung der endotrachealen Intubation. Die Intubationsvermeidung ist für die Akutmedizin der wichtigste Aspekt. In diesem Sinne wird NIV als Überbrückungs- und Ersatztherapie bei akutem respiratorischem Versagen [1-3], als Extubationshilfe zur Verkürzung einer laufenden invasiven Beatmung [4,5], und als Reintubationsprophylaxe nach erfolgter Extubation [6,7] eingesetzt.

Nach Jahren der Anwendung herrscht wenig Zweifel an der Effektivität dieser Beatmungsform [8-10]. Meta-Analysen bestätigen [11,12], dass NIV auch bei akutem respiratorischem Versagen Intubationsraten reduziert $[2,3,13]$, Beatmungsdauer verkürzt [1] und Mortalität senkt [1,2]. Dieser Behandlungsvorteil ist eindeutig erwiesen für Patienten mit akutem kardiogenem Lungenödem und Hyperkapnie infolge erschöpfter Atemmuskulatur [13 - 15], für akut exazerbierte COPD [16,17] und für selektionierte Krankheitsbilder mit hypoxämischem Atemversagen nach myeloablativer Therapie [18] oder solider Organtransplantation [19]. Der Behandlungsvorteil ist weniger eindeutig bei Patienten mit herkömmlichen Pneumonien, ARDS, Sepsis oder Trauma $[1-3,11,15,17,20]$. Obwohl NIV auch bei diesen Krankheitsbildern Intubationsrate, Beatmungslänge und Komplikationen senkt, bleibt die Mortalität meist unbeeinflusst [1-3,21,22]. Mit einer NIV-Versagerquote von 30-40\% muss gerechnet werden [23,24]. Die Aussichten auf Beatmungserfolg sinken grundsätzlich mit dem Schweregrad der akuten Erkrankung [1]. Auch scheinen NIV-Versager eine höhere Mortalität zu haben [1,24], was auf den Schweregrad der Erkrankung oder eine unbotmäßige Verzögerung der Intubation verweist. Dass NIV nicht für je- dermann geeignet ist, spiegelt auch die Tatsache wieder, dass a priori nur ein kleiner Prozentsatz der Akutpatienten für NIV qualifiziert [23]. Leider konnten verlässliche Prädiktoren für Erfolg oder Misserfolg bisher nicht gefunden werden [24,26-28].

In diesem Sinne überraschen auch nicht die Ergebnisse der jüngsten randomisiert-kontrollierten Multizenterstudie von Esteban [29]. Die Studie umfasste 221 Intensivpatienten, die wegen akuten respiratorischen Versagens mindestens 48 h invasiv beatmet worden waren und $48 \mathrm{~h}$ nach Extubation erneute Zeichen der respiratorischen Insuffizienz zeigten. Die Patienten wurden randomisiert zwei Therapiestrategien zugeordnet, einer konventionellen mit $\mathrm{O}_{2}$, Bronchodilatoren und Pulmonaltoilette, und einer alternativen mit NIV. Wegen einer höheren Mortalität im NIV-Arm (25 versus 15\% im konventionellen Arm) wurde die Studie vorzeitig abgebrochen. Die höhere Mortalität bezog sich hauptsächlich auf reintubierte Patienten im NIV-Arm (38\% versus $22 \%$ im konventionellen Arm, p=0,048). Obwohl sich die Gesamtreintubationsraten zwischen den Studienarmen nicht unterschieden (48\% im NIV-Arm, 51 \% im konventionellen Arm), war doch die Zeit zur Reintubation im NIV-Arm deutlich verlängert (12 h versus 2,5 h im konventionellen Arm).

Die Studie ist aus mehreren Gründen interessant. Zum einen bestätigt sie, dass neue Therapieansätze grundsätzlich auf objektivierbaren Behandlungsvorteil am klinischen Kollektiv geprüft werden müssen. Erst die systematische Anwendung kann klinische Einflussgrößen mit allen Wechselwirkungen erfassen, was unter Umständen dazu führt, dass vorteilhafte Maßnahmen in ihrer positiven Wirkung neutralisiert oder gar umgekehrt werden. Bezüglich der NIV scheinen zwei Umstände bedeutsam: zum einen scheint der die Atemmuskulatur entlastende Wirk- 
mechanismus bei rein hypoxämischem Versagen am Kernproblem vorbeizugehen. Zum zweiten besteht die Gefahr, durch ineffektive NIV-Versuche irreversiblen mikrozirkulatorischen Schäden Vorschub zu leisten. Dies wäre eine Erklärung für die höhere Mortalität im NIV-Versager-Arm.

Die Studie demonstriert aber auch, wie schwierig eine neutrale Interpretation klinischer Daten sein kann. Studienanalyse und Diskussion gehen nicht auf den Umstand ein, dass gut ein Viertel der Patienten im konventionellen Arm wegen progressiver Verschlechterung auf NIV „geswitched“ worden waren. Von diesen „rescue“ beatmeten Patienten musste nur ein Viertel, d.h. die Hälfte weniger als im nicht-rescue beatmeten Teil, intubiert werden. Auch die Mortalität der rescue-Beatmeten war um knapp ein Drittel weniger als im nicht-geswitchten Teil. Aus methodischer Sicht stellt sich die Frage, ob die rescue-Beatmeten des konventionellen Armes korrekterweise nicht als Therapieversager deklariert und dem reintubierten Teil des konventionellen Armes zugeordnet hätten werden müssen, oder als NIV-,.geswitchte" Patienten dem primären NIV-Beatmungsarm. Beides hätte die Studienergebnisse signifikant zu Gunsten der NIV geändert. Insofern besteht erheblicher Zweifel, ob mit der aktuellen Studie die Diskussion über den Stellenwert von NIV als Reintubationsprophylaxe tatsächlich beendet ist.

Sicher ist jedenfalls, dass der Einsatz von NIV in der Intensivmedizin nicht unproblematisch ist. Potenziell ist viel zu gewinnen, aber auch einiges zu verlieren. Restriktive Patientenselektion und kritische Indikationsstellung sind entscheidend für den $\mathrm{Er}$ folg. Wie auch der rechtzeitige Therapieabbruch, sollte sich kein erkennbarer Erfolg innerhalb weniger Stunden nach Beatmungsbeginn einstellen. Unter Berücksichtigung dieser Vorsichtmaßnahmen, und bei Gewährleistung maximaler Anwenderexpertise vor Ort, bleibt die NIV eine wertvolle und vielseitig einzusetzende Therapiemaßnahme auch in der Akutmedizin [8].

\section{Literatur}

${ }^{1}$ Antonelli M, Conti G, Rocco M et al. A comparison of noninvasive positive pressare ventilation and conventional mechanical ventilation in patients with acute respiratory failure. N Engl J Med 1998; 339: 429

2 Ferrer M, Esquinas A, Leon M et al. Noninvasive ventilation in severe hypoxemic respiratory failure. Am J Respir Crit Care Med 2003; 168: 1438

${ }^{3}$ Martin TJ, Hovis JD, Constantino JP et al. A randomized prospective evaluation of noninvasive ventilation for acute respiratory failure. Am J Respir Crit Care Med 2000; 161: 807

${ }^{4}$ Girault C, Daudenthun I, Chevron V et al. Noninvasive ventilation as a systematic extubation and weaning technique in acute-on-chronic respiratory failure: A prospective, randomized controlled study. Am J Respir Crit Care Med 1999; 160: 86

${ }^{5}$ Ferrer M, Esquinas A, Aranciba $\mathrm{F}$ et al. Noninvasive ventilation during persistant weaning failure: A controlled randomized trial. Am J Respir Crit Care Med 2003; 168: 70

${ }^{6}$ Kilger E, Briegel J, Haller M et al. Effects of noninvasive positive pressure ventilatory support in non-COPD patients with acute respiratory insufficiency after early extubation. Intensive Care Med 1999; 25: 1374
${ }^{7}$ Keenan SP, Powers C, McCormack DG et al. Noninvasive positive pressure ventilation for postextubation respiratory distress: a randomized controlled trial. JAMA 2002; 287: 3238

${ }^{8}$ International consensus conference in Intensive Care Medicine. Noninvasive positive pressure ventilation in acute respiratory failure. Am J Respir Crit Care Med 2001; 163: 283

${ }^{9}$ Mehta S, Hill NS. Noninvasive ventilation. Am J Respir Crit Care Med 2001; 163: 540

${ }^{10}$ Brochard L. Noninvasive ventilation in acute respiratory failure. JAMA 2002; 288 (8): 932 - 935

11 Peter JV, Moran JL, Phillips-Hughs J et al. Noninvasive ventilation in acute respiratory failure: a meta-analysis update. Crit Care Med 2002; 30: 555

${ }^{12}$ Lightowler JV, Wedzicha JA, Elliott MW et al. Non-invasive positive pressure ventilation to treat respiratory failure resulting from exacerbations of chronic obstructive disease: Cochrane systematic review and meta-analysis. BJM 2003; 326: 185

${ }^{13}$ Masip J, Betbese AJ, Paez J et al. Noninvasive pressure support ventilation versus conventional oxygen therapy in acute cardiogenic pulmonary edema: a randomized trial. Lancet 2000; 356: 2126

${ }^{14}$ Rusterholz T, Kempf J, Berton C et al. Noninvasive pressure support (NIPSV) with face mask in patients with cardiogenic pulmonary edema (ACPE). Intensive Care Med 1999; 25: 21

${ }^{15}$ Nava S, Carbone G, DiBattista $\mathrm{N}$ et al. Noninvasive ventilation in cardiogenic pulmonary edema. Am J Respir Crit Care Med 2003; 168: 1432

${ }^{16}$ Brochard L, Mancebo J, Wysocki M et al. Noninvasive ventilation for acute exacerbations of chronic obstructive pulmonary disease. N Engl J Med 1995; 333: 817

17 Wysocki M, Tric L, Wolff MA et al. Noninvasive pressure support ventilation in patients with acute respiratory failure: A randomized comparison with conventional therapy. Chest 1995; 107: 761

18 Hilbert G, Gruson D, Vargas F et al. Noninvasive ventilation in immunosuppressed patients with pulmonary infiltrates, fever, and acute respiratory failure. N Eng J Med 2001; 344: 481

${ }^{19}$ Antonelli M, Conti G, Buff M et al. Noninvasive ventilation for treatment of acute respiratory failure undergoing solid organ transplantation: a randomized trial. JAMA 2000; 283: 235

${ }^{20}$ Ambrosino N, Foglio K, Rubini F et al. Non-invasive mechnaical ventilation in acute respiratory failure due to chronic obstructive pulmonary disease: correlates for success. Thorax 1995; 50: 755

${ }^{21}$ Gonfalonieri M, Potena A, Carbone G et al. Acute respiratory failure in patients with severe community-acquired pneumonia. A prospective randomized evaluation of non-invasive ventilation. Am J Respir Crit Care Med 1999; 160: 1585

${ }^{22}$ Keenan SP, Kernerman PD, Cook DJ et al. Effect of noninvasive positive pressure ventilation on mortality in patients admitted with acute respiratory failure: a meta-analysis. Crit Care Med 1997; 25: 1685

${ }^{23}$ Antonelli M, Conti G, Moro ML et al. Predictors of failure for noninvasive positive pressure ventilation in patients with acute hypoxemic respiratory failure: a multi-center study. Intensive Care Med 2001; 27: 1718

${ }^{24}$ Esteban A, Anzueto A, Frutos F et al. Characteristics and outcomes of adult patients receiving mechanical ventilation: a 28-day international study. JAMA 2002; 287: 245

${ }^{25}$ Wood KA, Lewis L, Harz B von et al. The use of noninvasive positive pressure ventilation in the emergency department: results of a randomized clinical trial. Chest 1998; 113: 1339

${ }^{26}$ Hess D. Noninvasive positive pressure ventilation: predictors of success and failure for adult acute care application. Respir Care 1997; 42: 424

27 Sinuff T, Keenan SP. Clinical practice guidelines for the use of noninvasive positive pressure ventilation in COPD patients with acute respiratory failure. J Crit Care 2004; 19: 82

${ }^{28}$ Anton A, Guell R, Gomez J et al. Predicting the result of noninvasive ventilation in severe acute exacerbations of patients with chronic airflow limitation. Chest 2000; 117: 828

${ }^{29}$ Esteban A, Frutos-Vivar F, Ferguson ND et al. Noninvasive positive pressure ventilation for respiratory failure after extubation. $\mathrm{N}$ Engl J Med 2004; 350: 2452 